\title{
SFPQ promotes an oncogenic transcriptomic state in melanoma
}

\author{
O. $\mathrm{Bi}^{1}$, C. A. Anene ${ }^{2}$, J. Nsengimana $\mathbb{D}^{3}$, M. Shelton ${ }^{1}$, W. Roberts ${ }^{4}$, J. Newton-Bishop ${ }^{5}$ and J. R. Boyne $\mathbb{D}^{1 凶}$ \\ (c) Crown 2021
}

The multifunctional protein, splicing factor, proline- and glutamine-rich (SFPQ) has been implicated in numerous cancers often due to interaction with coding and non-coding RNAs, however, its role in melanoma remains unclear. We report that knockdown of SFPQ expression in melanoma cells decelerates several cancer-associated cell phenotypes, including cell growth, migration, epithelial to mesenchymal transition, apoptosis, and glycolysis. RIP-seq analysis revealed that the SFPQ-RNA interactome is reprogrammed in melanoma cells and specifically enriched with key melanoma-associated coding and long non-coding transcripts, including SOX10, AMIGO2 and LINC00511 and in most cases SFPQ is required for the efficient expression of these genes. Functional analysis of two SFPQ-enriched InCRNA, LINC00511 and LINC01234, demonstrated that these genes independently contribute to the melanoma phenotype and a more detailed analysis of LINC00511 indicated that this occurs in part via modulation of the miR-625$5 \mathrm{p} /$ PKM2 axis. Importantly, analysis of a large clinical cohort revealed that elevated expression of SFPQ in primary melanoma tumours may have utility as a prognostic biomarker. Together, these data suggest that SFPQ is an important driver of melanoma, likely due to SFPQ-RNA interactions promoting the expression of numerous oncogenic transcripts.

Oncogene (2021) 40:5192-5203; https://doi.org/10.1038/s41388-021-01912-4

\section{INTRODUCTION}

At the molecular level, melanoma comprises a heterogeneous group of disorders that harbour distinct aberrations in diverse cellular processes [1-3]. Such heterogeneity suggests that multiple mechanisms are involved in disease aetiology, and this is reflected in the contribution of both different mutations and differential gene and protein expression associated with melanoma transformation and progression [4].

Splicing factor proline- and glutamine-rich (SFPQ) is a multifunctional DNA- and RNA-binding protein that regulates numerous cellular mechanisms, including RNA-processing, transcription, DNA damage response and innate immunity [5-9]. Aberrant SFPQ function is associated with the aetiology of neurodegenerative disorders [10-12] and colorectal [13], hepatocellular [14], renal [15], Chronic Myeloid Leukaemia [16] and prostate cancer [17]. Intriguingly, it appears that SFPQ-long non-coding RNA (IncRNA) interactions are responsible for pathological changes in numerous cancers $[14,15,18]$ including breast $[19,20]$ and ovarian tumours [21]. Despite comprising a family of up to 90,000 transcripts [22], the majority of IncRNAs are incompletely annotated and remain poorly understood at the functional level. However, dysregulated expression of IncRNA has been shown to correlate with poor outcome across a range on neoplasms, with increasing numbers of IncRNA being implicated in driving metastasis [23-25].

Here we investigated the role of SFPQ in melanoma cells and showed that expression of SFPQ contributes to melanoma cell growth, migration, apoptosis, glycolysis, and epithelial to mesenchymal transition (EMT). Investigation and interrogation of the SFPQ-RNA interactome in primary melanocytes compared with melanoma cells via RNA-immunoprecipitations (RIP)-seq revealed a dramatic reprogramming in melanoma that favoured coding and non-coding transcripts associated with the promotion of metastatic disease. Furthermore, knockdown of SFPQ led to a significant decrease in many of these enriched transcripts, including the IncRNA, LINC00511, which we showed contributes to the melanoma cell phenotype. Finally, analysis of our melanoma patient data revealed that high levels of SFPQ expression in tumours negatively correlate with patient survival, suggesting that SFPQ may have utility as a prognostic biomarker.

\section{RESULTS \\ SFPQ knockdown decelerates cancer cell phenotype in melanoma}

To investigate the role of SFPQ in melanoma, we analysed SFPQ protein expression in untransformed primary human melanocytes (PM) alongside a range of melanoma cell lines. As can be seen in Fig. 1a, we observed a significant increase in SFPQ expression in each melanoma cell line compared with PM. SFPQ has been identified as an essential melanoma gene in a CRISPR genomewide loss of function screen [26], and analysis of this dataset revealed that melanoma cell lines $(n=46)$ show high dependency (DEMETER2 scores) on SFPQ regardless of its expression (i.e., poor correlation between dependency score and expression levels) (Fig.

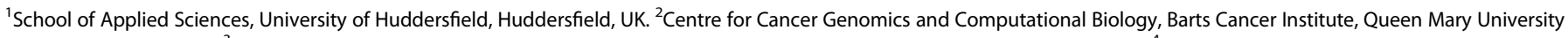

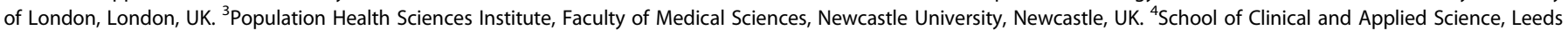
Beckett University, Leeds, UK. ${ }^{5}$ University of Leeds School of Medicine, Leeds, UK. ${ }^{凶}$ email: j.r.boyne@hud.ac.uk
}

Received: 5 November 2020 Accepted: 17 June 2021 
a

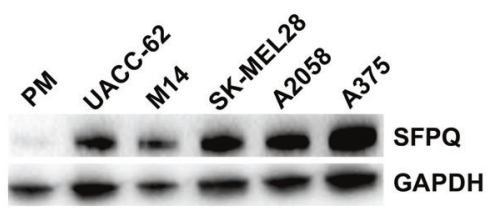

b

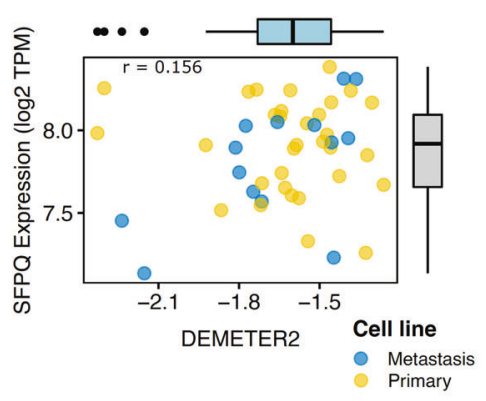

C
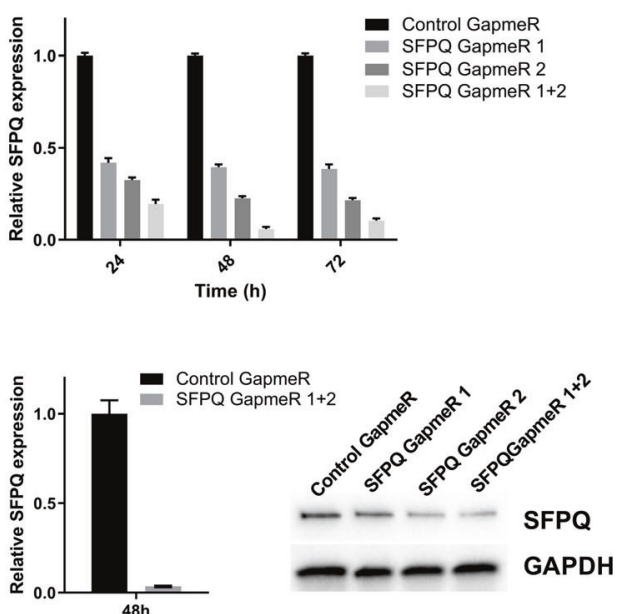

d

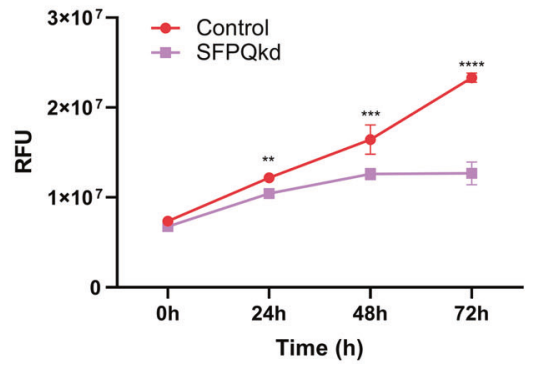

e

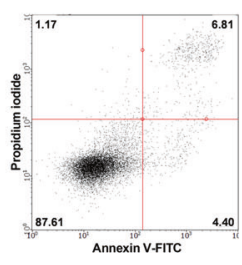

f

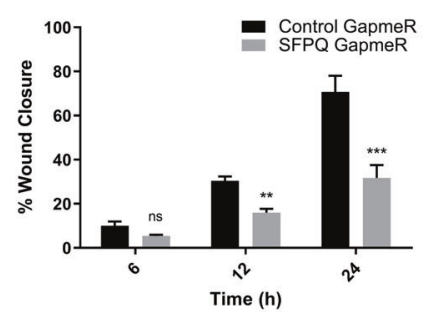

g
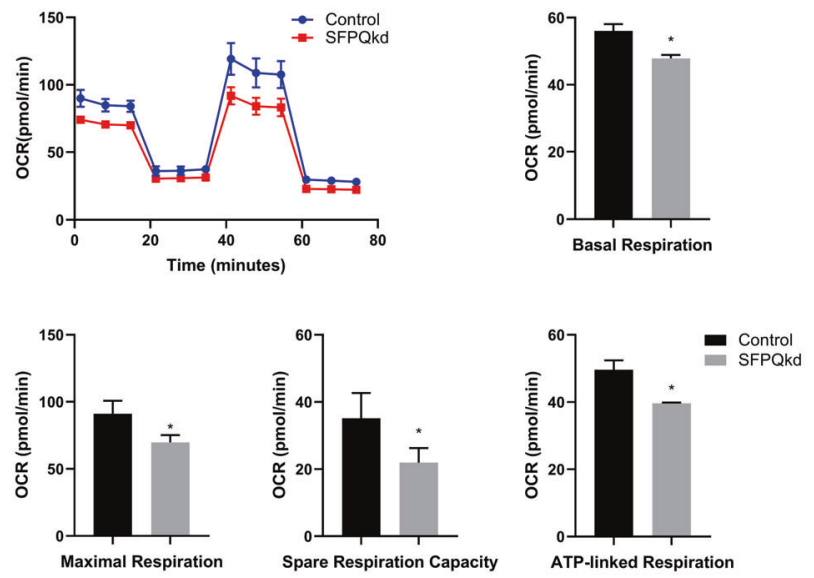

h
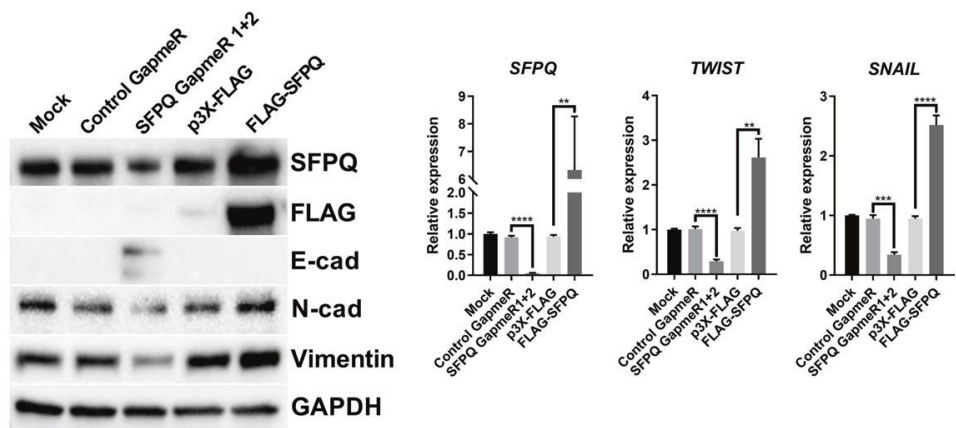

- Control
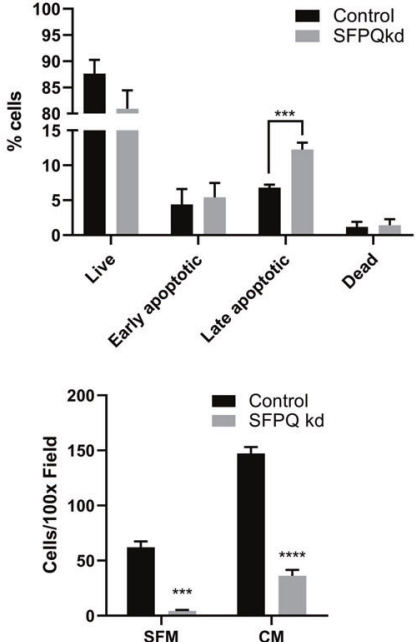
Fig. 1 SFPQ expression promotes the cancer phenotype in melanoma. a Total protein was isolated from primary melanocytes (PM) and melanoma cell lines prior to analysis of SFPQ expression via immunoblot, $n=3$. b SFPQ dependency score ( $x$-axis) was compared with SFPQ expression levels ( $y$-axis) in melanoma cell lines. Within the plot, lower DEMETER2 scores correspond to more dependency on SFPQ, and positive values indicate no dependence. c Total RNA was extracted from A2058 cells transiently transfected with SFPQ-specific GapmeR or control for 24, 48 and $72 \mathrm{~h}$ prior to generation of CDNA and analysis of SFPQ expression via qPCR, $n=3$. d Viable growth of A2058 cells transiently transfected with the indicated GapmeR and cultured for 24,48 and $72 \mathrm{~h}$ was determined using CellTitre-Glo, $n=3$. e A2058 cells were transiently transfected with SFPQ-specific GapmeR or control and cultured for $48 \mathrm{~h}$ prior to staining with Annexin V-FITC and Propridium iodide and analysis via FACS, $n=3$. f A2058 cells were transfected with SFPQ-specific GapmeR or control and cultured for $48 \mathrm{~h}$ prior to analysis of cell migration via wound healing (left panel) and transwell (right panel) assay, $n=3$. g A2058 cells were transfected with SFPQ-specific GapmeR or control and cultured for $48 \mathrm{~h}$ prior to analysis of cellular respiration using the Cell Mito Stress Test kit, $n=3$. $\mathbf{h}$ A2058 cells were transiently transfected with SFPQ-specific GapmeRs, pFLAG-SFPQ, or corresponding scramble GapmeR and parent vector controls and cultured for $48 \mathrm{~h}$, prior to isolation of total protein and RNA and analysis of indicated EMT markers via western blot and qRT-PCR, respectively, $n=3$.

significant decrease in basal respiration, maximal respiration, ATP production and spare respiration capacity, suggesting impaired OXPHOS in cells with decreased expression of SFPQ. Finally, we investigated the impact of SFPQ knockdown and overexpression on several EMT markers and observed a decrease in the expression of mesenchymal markers following knockdown of SFPQ and an increase in cells where SFPQ expression was increased (Fig. 1h). Together, these data demonstrate that knockdown of SFPQ in melanoma cells results in a wide-ranging attenuation of the cancer cell phenotype.

\section{Comparative analysis of SFPQ-RNA interactome in primary melanocytes and melanoma cells}

Given data demonstrating a role for SFPQ in melanoma cell phenotype, we were keen to characterise the SFPQ-RNA interactome in $\mathrm{PM}$ and establish if this was reprogrammed in melanoma cells. To this end, PM and A2058 melanoma cells were lysed, and RIP performed using either an SFPQ-specific antibody or IgG-antibody isotype control (Fig. S1A), followed by analysis via RNA-seq. Initially, we identified all transcripts that were (i) significantly enriched with SFPQ in PM compared with A2058 cells; (ii) enriched with SFPQ in both PM and A2058 cells; (iii) significantly enriched with SFPQ in A2058 cells compared with PM (Fig. 2a). Most SFPQ-enriched transcripts comprised mRNAs, with 8124 unique peaks called, however, further analysis revealed 345 SFPQ-IncRNA interactions across both cell backgrounds (Fig. 2b). We observed significant differential enrichment of both mRNA and IncRNA transcripts between PM and A2058 cells and while the RNA biotype with shared enrichment included significantly more genes, the non-coding RNA (ncRNA) biotype was more significant in A2058, suggesting specific reprogramming of SFPQ-IncRNA interactions in melanoma. (Fig. 2c). To determine if baseline expression of these differentially enriched genes in PM and A2058 transcriptomes was influencing SFPQ interaction, whole-cell RNA expression profiles were integrated with RIP-Seq data with unsupervised clustering. We observed no association between whole-cell RNA transcript abundance and the specificity of the SFPQ-RNA interactions (Fig. 2d and Fig. S1B). Gene ontology (GO) analysis was then performed to identify enriched biological processes for PM-specific and A2058-specific SFPQ-mRNA interactors, respectively. Interestingly, PM-specific targets were significantly enriched for GO terms broadly associated with extracellular matrix (ECM) organisation, whereas A2058-specific targets were significantly associated with $\mathrm{GO}$ terms relating to the positive regulation of the cell cycle (Fig. 2e).

Given the widely documented relationship between SFPQ and IncRNAs in several cancers, including melanoma, SFPQ interactors were also ranked according to their PM- and A2058-specificity (Fig. $2 \mathrm{f}, \mathrm{g}$ ). Discerning the functional importance of enriched IncRNA gene lists from RNA-seq studies such as the one reported here is problematic, as the vast majority of IncRNAs remain functionally uncharacterised. However, it is interesting to note that the most PM-specific SFPQ-IncRNA interactors included genes associated with tumour suppressor function, such as EMX2OS and FENDRR $[27,28]$, whereas the most A2058-specific SFPQ-IncRNA interactors comprise genes widely reported as oncogenic, such as LINC00511 [29-31]. We next sought to validate the top hits from our A2058 RIP-seq data in additional melanoma cell lines. To this end, SFPQ RIPs were performed using cell lysate isolated from the M14 and UACC-62 melanoma cell lines and enrichment with SFPQ assessed via qRT-PCR. As can be seen in Fig. $2 \mathrm{~h}$, the top mRNA and IncRNA targets enriched with SFPQ in the A2058 dataset were also significantly enriched with SFPQ in M14 and UACC-62 cells, whereas transcripts that were not present in our RIP-seq dataset did not exhibit significant enrichment with SFPQ. Collectively, these data show that compared with PM, SFPQ-RNA interactions in melanoma cells are reprogrammed and skewed towards oncogenic coding and non-coding transcripts.

\section{SFPQ-enriched melanoma-specific IncRNA contribute to the cancer phenotype}

RIP-seq analysis identified 137 IncRNA that is enriched with SFPQ in A2058 melanoma cells. To establish if any novel melanomaspecific SFPQ-enriched IncRNA were functionally important for the cancer phenotype, we opted to investigate two SFPQ-enriched genes, LINC00511 and LINC01234, which have recently been implicated in the progression of cancer, but to date have not been associated with melanoma. Initially, A2058 cells were transfected with either control, LINC00511- or LINC01234-specific GapmeR ASOs and knockdown of target transcript levels confirmed via qRT-PCR (Fig. 3a). We observed a marked reduction in LINC00511depleted viable cell growth over $72 \mathrm{~h}$ and a lesser, but still significant reduction in growth kinetics following knockdown of LINC01234 (Fig. 3b). Interestingly, caspase $3 / 7$ activity was significantly increased following knockdown of LINC00511, but not in LINC01234-depleted cells (Fig. 3c). In contrast, we observed a significant decrease in cell migration following knockdown of either LINC00511 or LINC01234 (Figs. 3d, e). Finally, whereas knockdown of LINCO1234 had little impact on cellular respiration, LINC00511-depleted A2058 cells exhibited nominal but significant decreases for basal and maximal respiration, ATP-linked respiration, and spare respiration capacity (Fig. 3f).

\section{LINC00511-miR-625-5p-PKM2 axis promotes glycolysis in melanoma}

Given the pronounced effect of LINC00511 knockdown in A2058 cells on cancer cell phenotype, we decided to investigate this gene further. To this end, publicly available Ago2-CLIP data [32] was analysed to identify miRNAs that interact with LINC00511 and miR-625-5p identified as the top interactor (Fig. 4a), an observation supported by several recent articles demonstrating that LINC00511 directly sponges miR-625-5p in renal, gastric and lung cancer [33-35]. To investigate if LINCO0511 is functioning to sponge miR-625-5p in melanoma, LINC00511 was knocked down in A2058 cells, as described above, and miR-625-5p expression analysed via miRCURY LNA miRNA PCR Assay. As can be seen in 
a

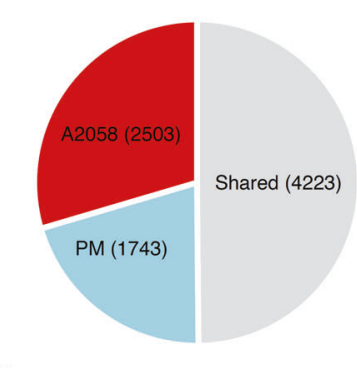

b

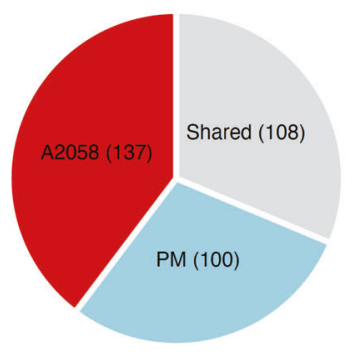

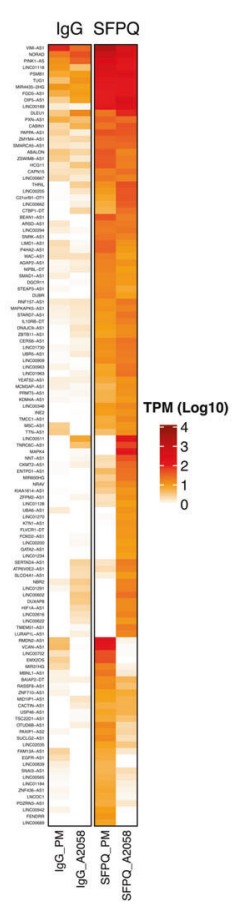

d

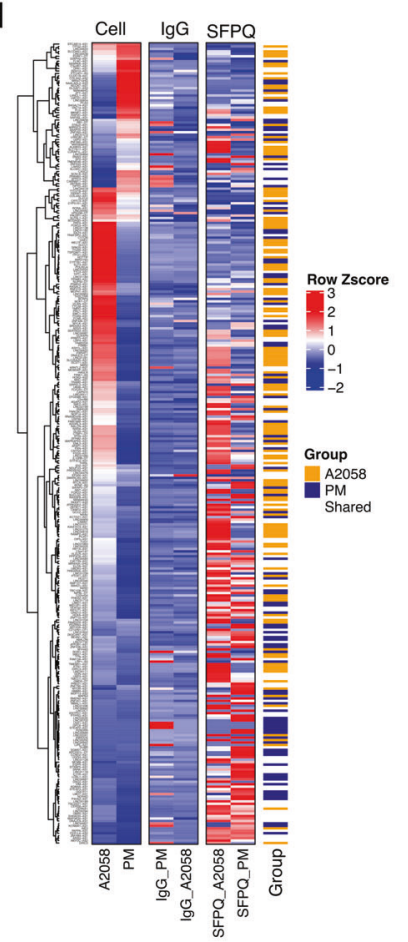

f

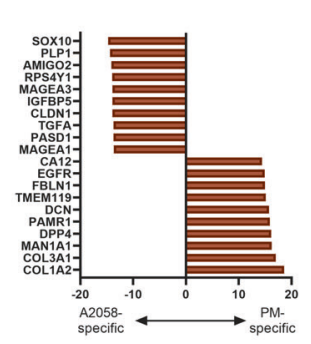

g

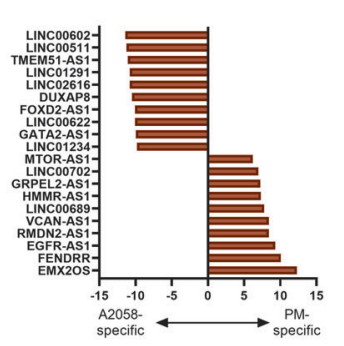

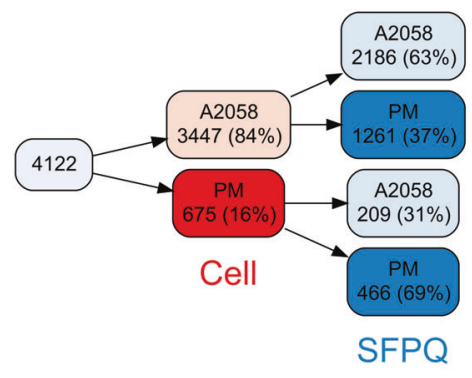
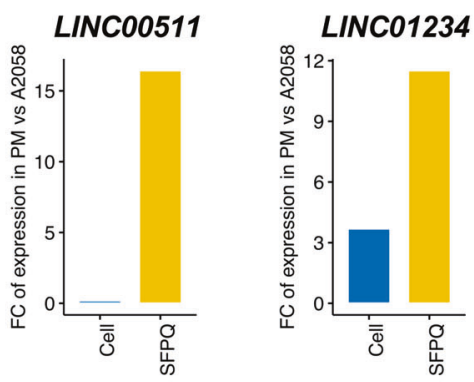

e

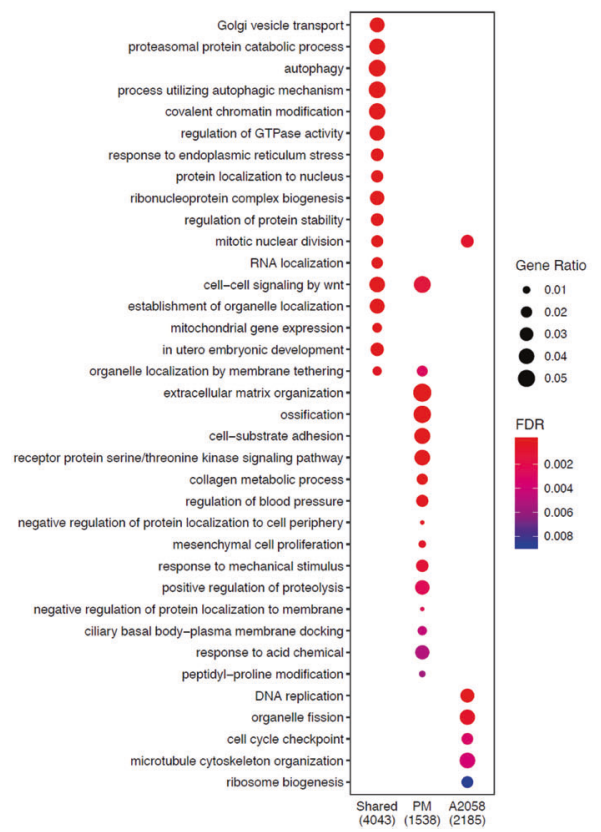

h
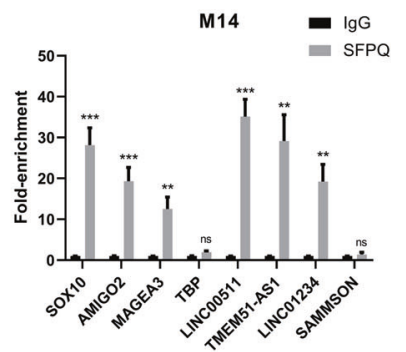

UACC-62 $=\lg$

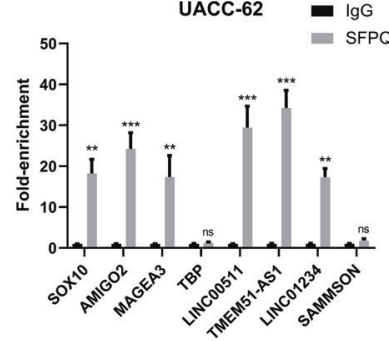

Fig. 4b, depletion of LINC00511 led to a significant increase in miR625-5p transcript level, compared with control and knockdown of LINC01234, suggesting that LINC00511 may sponge miR-625-5p in melanoma cells. Recently, miR-625-5p was reported to target the PKM2 isoform of pyruvate kinase in melanoma to attenuate glucose consumption. To explore if LINC00511 knockdown led to a decrease in the expression of PKM2, LINC00511-depleted A2058 melanoma cells were analysed via qRT-PCR and a significant decrease in PKM2 expression was observed, whereas no decrease in PKM2 expression was seen in either SFPQ- or LINC01234depleted cells (Fig. 4c and Fig. S2). Consistently, analysis of the
TCGA-melanoma dataset demonstrated that amplification of LINC00511 significantly increased the expression of PKM2 (Fig.4d). To investigate if the decrease in PKM2 transcripts was due to impaired LINC00511-mediated sponging of miR-625-5p, LINC00511-depleted cells were co-transfected with miR-625-5pspecific inhibitor or scramble control and PKM2 expression assessed, as described above. As can be seen in Fig. 4e, transfection with miR-625-5p inhibitor restored PKM2 transcript abundance following LINC00511 knockdown. Next, we utilised Seahorse XF analysis to determine if inhibition of miR-625-5p rescued the decrease in OCR in LINC00511-depleted cells. Data in 
Fig. 2 SFPQ-RNA interactome is reprogrammed in A2058 melanoma cells. a Pie chart depicts the proportion of all interaction RNA transcripts shared between PM and A2058, specific to PM or specific to A2058. b Pie chart depicts the proportion of non-coding interacting RNA transcripts shared between PM and A2058, specific to PM or specific to A2058. c Heatmap of expression profile of all identified IncRNA interactors across IgG control and SFPQ-IP. Within the heatmap, orange colour indicates enrichment in SFPQ-IP compared to IgG and white colour indicates no enrichment. d Heat map of relative expression profile of identified IncRNA interactors across whole-cell RNA (A2058 and PM), IgG control and SFPQ-IP. Within the heatmap, red colour indicates high expression relative to whole-cell RNA and blue colour indicates low expression (left panel). Distribution plots SFPQ-specific interactions against cell-type expression levels. Within the plot, the left panel (white box) is the total number of SFPQ interactors, middle panel (Cell) indicates the proportion of SFPQ interactors in terms of cell-specific expression. The right panel (SFPQ) shows the distribution of SFPQ-specific interactions by cell type (upper right panel). A2058 and PM data were analysed to determine the fold-increase in relative cell expression compared with fold-increase in relative SFPQ-enrichment for the A2058-specific SFPQenriched InCRNAs, LINC00511 and LINC01234 (lower right panel). e Cluster comparison plot of enriched biological processes for transcripts shared between PM and A2058, specific to PM or specific to A2058. Gene ratio genes in ontology/total gene set and FDR false discovery rate. f SFPQ-enriched mRNA transcripts were ranked based on the specificity of enrichment in either PM or A2058 melanoma cells. g SFPQ-enriched IncRNA transcripts were ranked based on the specificity of enrichment in either PM or A2058 melanoma cells. $\mathbf{h}$ SFPQ-RNA interactions identified in A2058 via RIP-seq were confirmed via RNA-IP and qRT-PCR in M14 and UACC-62 melanoma cells, $n=3$.

Fig. $4 \mathrm{f}$ show that co-transfection of LINC00511 and miR-625-5p inhibitor led to a partial but significant rescue of the diminished basal and maximal OCR observed following LINC00511 knockdown. Together, these data demonstrate that expression of LINC00511 in melanoma regulates the miR-625-5p/PKM2 axis, which may explain LINC00511-dependent changes to OCR in A2058 melanoma cells.

\section{SFPQ regulates the expression of RNA interactors}

To determine if SFPQ regulates the transcript levels of interacting RNAs in melanoma, SFPQ expression was knocked down in A2058 cells and expression of several SFPQ-enriched mRNA and IncRNA genes determined via qRT-PCR. As can be seen in Fig. 5a, knockdown of SFPQ led to a significant decrease in the transcript abundance of several, but not all, SFPQ-enriched RNAs. Specifically, we observed approximately two-fold reduction in SOX10 and MAGEA3 transcript levels, but no reduction in the levels of $A M I G O 2$ or any of the three reference genes used to normalise qRT-PCR data (GAPDH, RPS13 and $T B P$ ). Interestingly, we also observed significant reductions in the transcript abundance of LINC00511, LINC01234 and TMEM51-AS1, which represented the top three SFPQ-enriched IncRNA genes by cell type specificity, however, we did not detect any change in the expression of the melanoma-specific IncRNA, SAMMSON, which was not enriched with SFPQ in our RIP-seq dataset. Similarly, overexpression of SFPQ resulted in significant increases to LINC00511 and LINC01234 transcript levels, but no change in the levels of SAMMSON (Fig. S3). To assess if observed changes in SFPQ-enriched transcript levels following knockdown of SFPQ were related to posttranscriptional regulation, SFPQ was depleted and RNA Pol II activity inhibited via treatment with actinomycin $D$, prior to analysis of target gene transcript levels over a $6 \mathrm{~h}$ period. Only SOX10 and TMEM51-AS1 exhibited decreased stability in SFPQ-depleted cells (Fig. 5b). To gain a better understanding of how SFPQ might be regulating the expression of enriched RNAs, SFPQ-enriched transcript regions were identified and annotated, and Integrative Genomics Viewer utilised to visualise coverage of the reads across the identified genes. Global analysis of SFPQ peaks revealed a similar profile in PM and A2058 melanoma cells, with most binding occurring at exon-intron boundaries and towards the $3^{\prime}$ end of transcripts (Fig. $5 \mathrm{c}$ and Fig. S4). Together, these data demonstrate that SFPQ contributes to the efficient expression of several melanoma-associated oncogenic transcripts in a predominantly RNA stability-independent manner, and by doing so contributes to the maintenance of a 'cancer transcriptomic state' in melanoma cells, in vitro.

SFPQ is a potential prognostic biomarker for melanoma Analysis of data detailing SFPQ expression in normal skin compared with nevi, primary and metastatic melanoma (GSE46517) was carried out and revealed that SFPQ expression is significantly increased in metastatic tumours compared with control and primary tumour samples (Fig. 6a). However, SFPQ expression groups (stratified by expression quantiles) were not associated with patient survival in the TCGA-melanoma dataset (Fig. 6b). To investigate if SFPQ expression groups in primary melanoma tumours were prognostic for survival, we utilised our previously published transcriptomic data from the primary melanomas of 703 patients, which comprises part of the Leeds Melanoma Cohort (LMC) [36]. Analysis of this cohort revealed that primary tumour expression of SFPQ significantly predicted patient survival with reduced survival for patients in the top three quartiles compared with the lowest quartile (Fig. 6c). Unfortunately, the HT12.4 array utilised does not contain probes for any of the melanoma-specific SFQP-enriched IncRNA described in this study, preventing analysis of LINC00511 and LINC01234 expression and patient survival in primary melanoma. Although less relevant, two PM-specific SFPQ IncRNA interactors were available for analysis in the LMC cohort and interestingly their expression was positively associated with patient survival (Fig. S5). To determine if SFPQ expression correlated with expression of LINC00511 and LINC01234 in melanoma tumours, we analysed TCGA-melanoma datasets on the GEPIA2 web portal [37] and found significant correlation between the expression of SFPQ and LINC00511 $(r=0.14, p=0.0027)$, and SFPQ and LINC01234 $(r=$ $0.24, p=2.2^{-07}$ ) (Fig. $6 \mathrm{~d}$ ), suggesting that these IncRNAs are positively associated with SFPQ. Together, these data demonstrate that increased expression of SFPQ in primary melanoma is associated with decreased melanoma patient survival and that SFPQ expression may have some utility as prognostic biomarker in primary melanoma.

\section{DISCUSSION}

The precise role of SFPQ in cancer is complex and appears to be tissue specific. For example, in breast and prostate cancer SFPQ appears to be an important driver of the cancer phenotype via post-transcriptional regulation of key genes such as oestrogen receptor a (ERa) and androgen receptor (AR), respectively $[17,20]$. Whereas in colorectal, lung and renal cancer SFPQ has been reported to act as a transcriptional repressor that serves to dampen proto-oncogene expression, a function that is frequently derailed via the overexpression and binding of oncogenic IncRNAs, such as MALAT-1 [38] and SANT1 [15]. The role of SFPQ in melanoma is less clear, with a single article suggesting SFPQ attenuates RAB23 expression via binding of the InCRNA, LLME23 [39]. Interestingly, we did not detect the LLME23 transcript in our A2058 RIP-seq dataset. Indeed, the LLME23 transcript remains incompletely annotated and is unlisted as a IncRNA in both the NCBI 109.20200815 and Ensembl 101 genome releases, casting some doubt on the nature of this gene. 
a

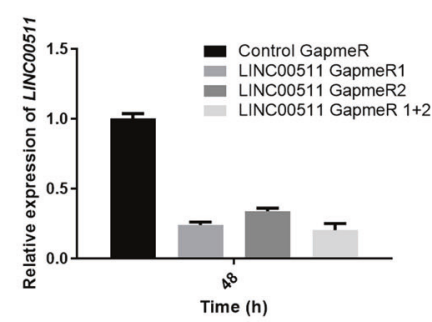

b

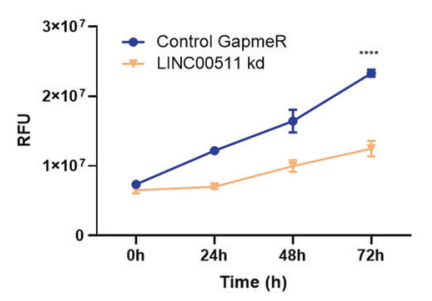

e
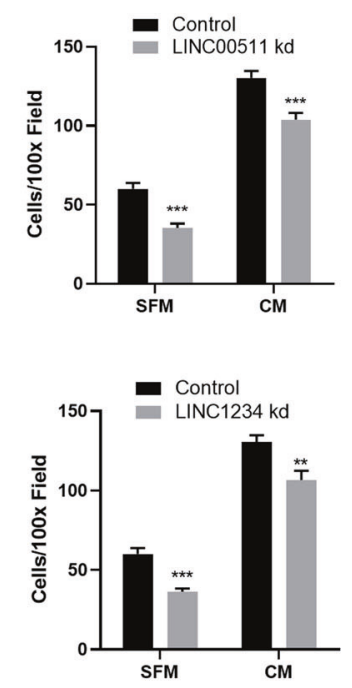

f

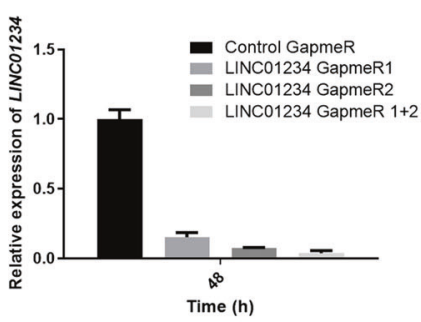

C

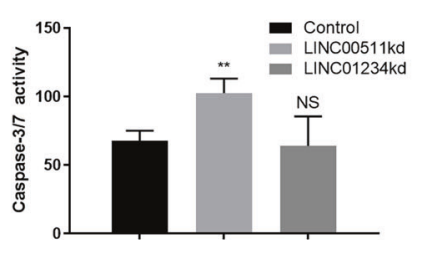

d
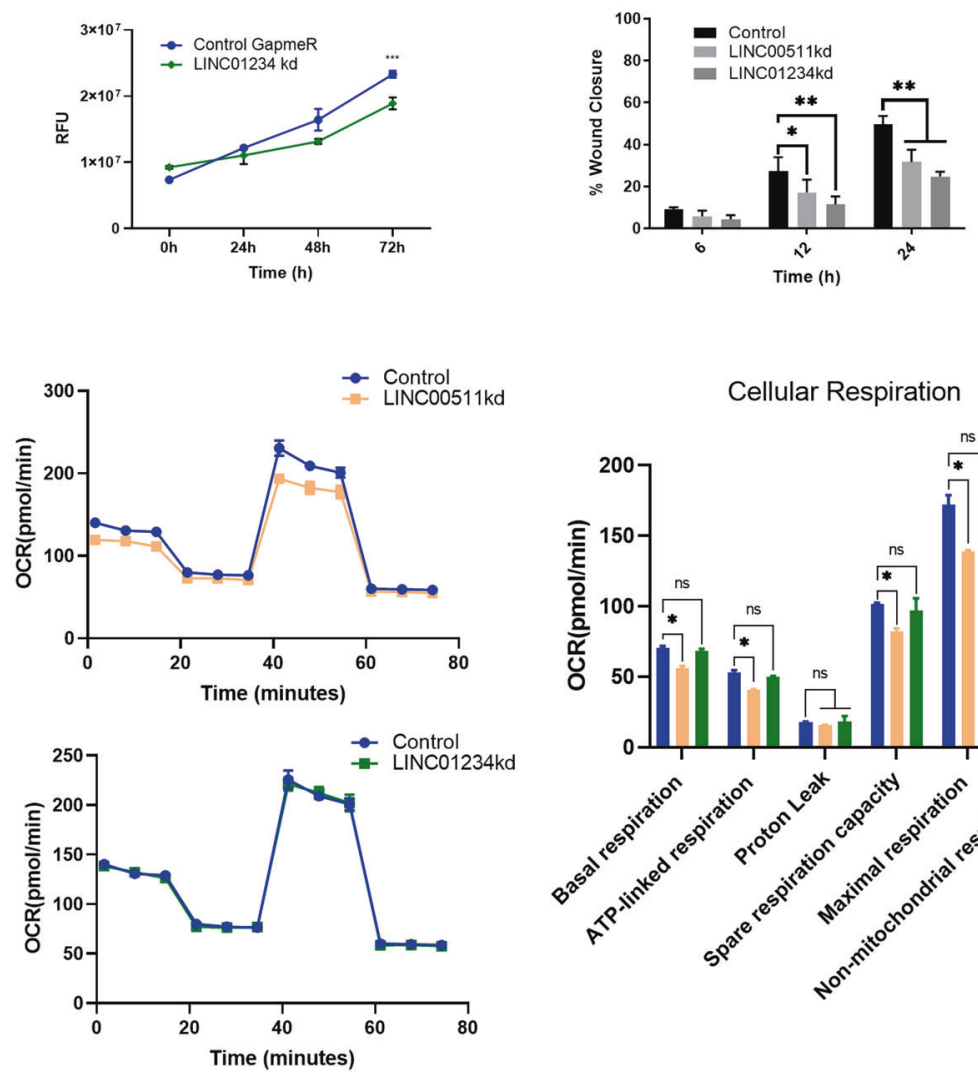

Cellular Respiration

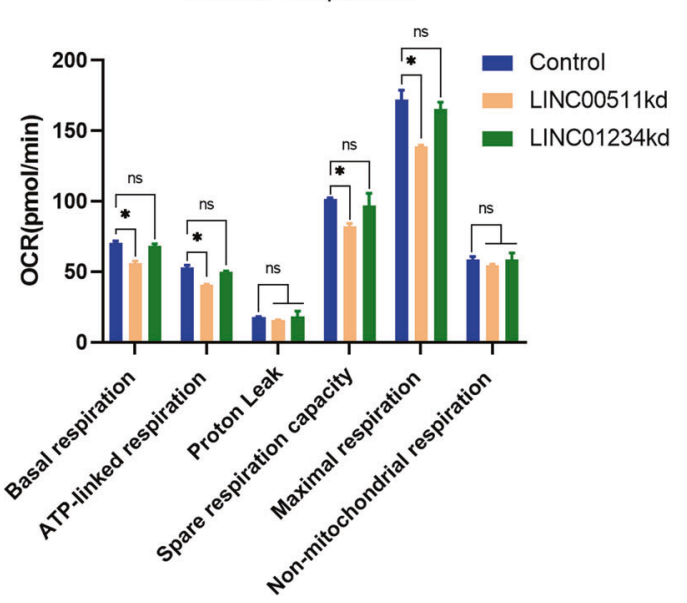

Fig. 3 LINC00511 and LINC01234 contribute to the cancer phenotype in A2058 melanoma cells. a Total RNA was extracted from A2058 cells transiently transfected with the indicated GapmeR for $48 \mathrm{~h}$ prior to generation of cDNA and analysis of target gene expression via qPCR, $n=3$. b Viable growth of A2058 cells transiently transfected with the indicated GapmeR and cultured for 24, 48 and $72 \mathrm{~h}$ was determined using CellTitre-Glo, $n=3$. c A2058 cells were transiently transfected with the indicated GapmeR and cultured for $48 \mathrm{~h}$ prior to addition of the Caspase-Glo ${ }^{\otimes} 3 / 7$ reagent. Plates were incubated at room temperature for $30 \mathrm{~min}$ and luminescence recorded, $n=3$. d A2058 cells were transfected with the indicated GapmeR and cultured for $48 \mathrm{~h}$ prior to analysis of cell migration via wound healing assay, $n=3$. e A2058 cells were transfected with the indicated GapmeR and cultured for $48 \mathrm{~h}$ prior to analysis of cell migration via transwell assay, $n=3 \mathbf{f} A 2058$ cells were transfected with the indicated GapmeR and cultured for $48 \mathrm{~h}$ prior to analysis of cellular respiration using the Cell Mito Stress Test kit, $n=3$.

Data in Fig. 1b-f suggest that the net functional output of SFPQ expression in melanoma cell lines is oncogenic. This hypothesis is supported by studies in hormone-refractory prostate cancer, where increased SFPQ expression has been reported to drive expression of $A R$ transcripts, in addition to other key driver genes, to promote disease progression (17). RIP-seq analysis demonstrated that the SFPQ-RNA interactomes of PM and melanoma cells diverged significantly and, crucially, in a manner that was not simply associated with relative transcript abundance (Fig. $2 \mathrm{c}$ ), which can be a limitation of RIP-seq, suggesting that these differences were due to reprogramming. Interestingly, gene-level analysis of SFPQenriched mRNA transcripts exclusively associated with PM revealed several targets that are frequently downregulated in melanoma and specifically associated with ECM architecture (COL1A2, COL3A1, Decorin, and Fubilin-1) (40), in addition to the proapoptotic tumour suppressor gene, DPP4, which has been reported as a suppressor of metastatic progression in melanoma [40]. In contrast, the top SFPQ-enriched mRNA transcripts in melanoma cells were generally associated with disease progression, such as AMIGO2, MAGEA3, MAGEA1, and the transcription factor, SOX10 [41-45].

A similar story emerged when we compared IncRNA enriched with SFPQ in PM and melanoma cell backgrounds. The SFPQenriched IncRNA transcripts most specific to PM were EMX2OS and FENDRR, the latter reported to function as a tumour suppressor in most cancers, including melanoma [27, 46-49]. The function of $E M X 2 O S$ in cancer differs depending on cell background $[28,50]$. 


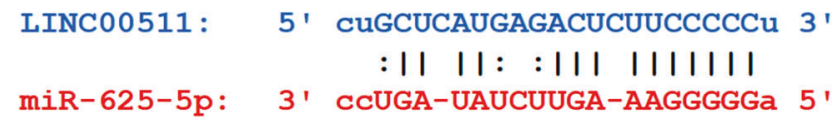

b

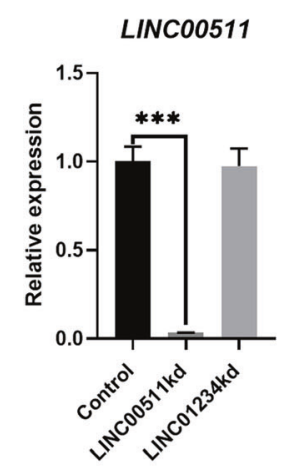

C
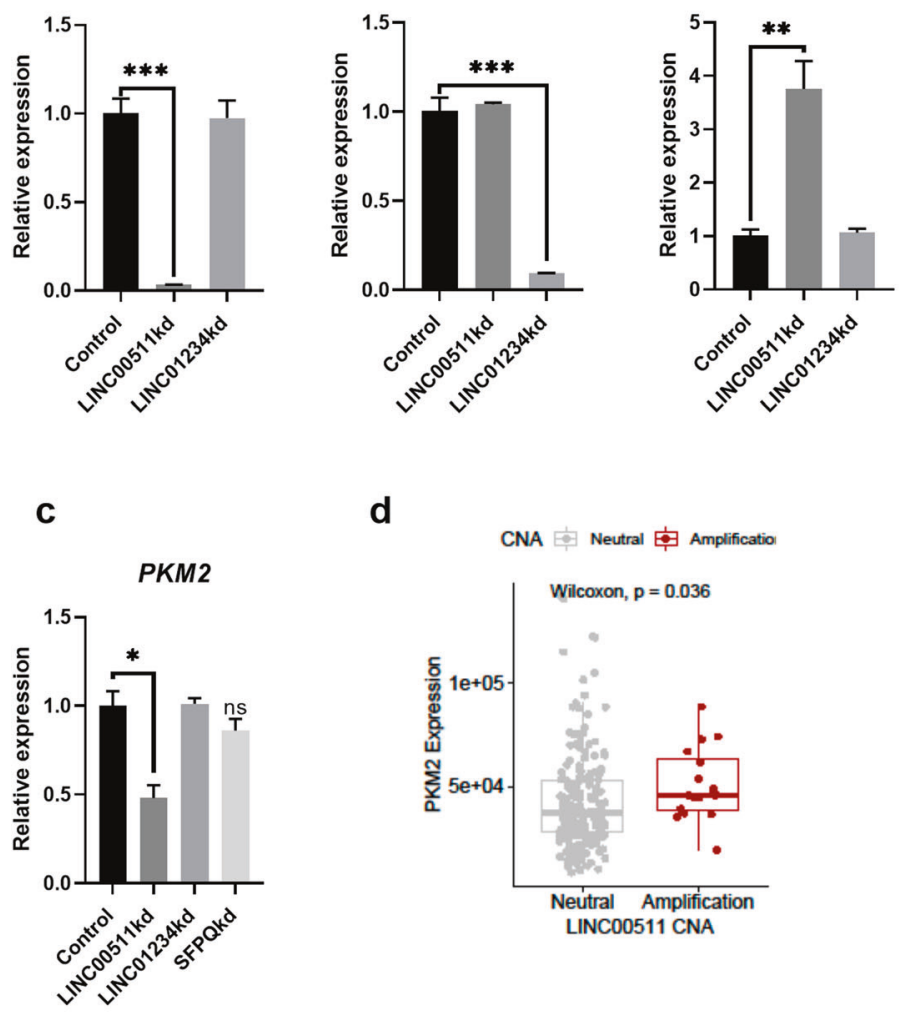

d CNA Neutral 审 Amplificatio

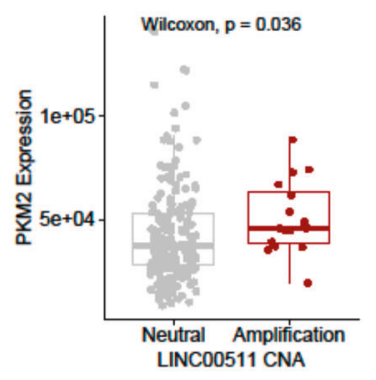

e

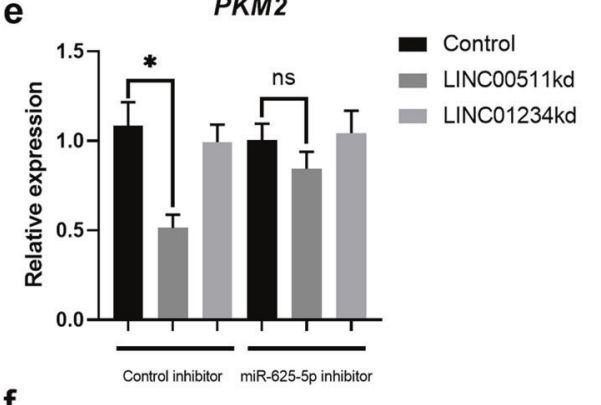

f

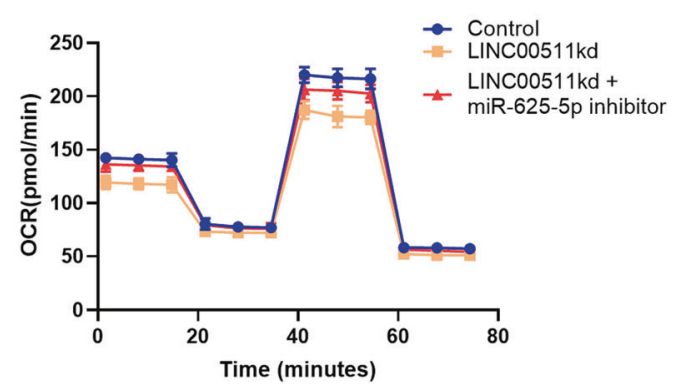

Cellular Respiration

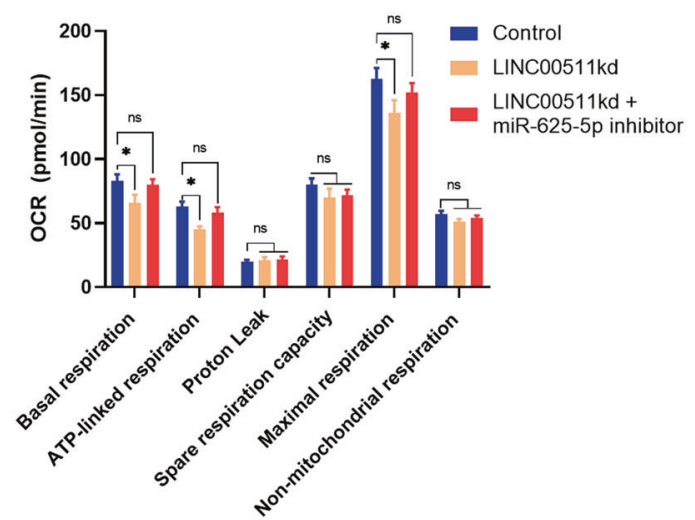

Fig. 4 LINC00511/miR-625-5p/PKM2 axis regulates cellular respiration in A2058 melanoma cells. a Analysis of HITS-CLIP-seq data identified miR-625-5p as a LINC00511 interaction partner. b Total RNA was extracted from A2058 cells transiently transfected with the indicated GapmeR for $48 \mathrm{~h}$ prior to generation of CDNA and analysis of target gene expression via qPCR (LINC00511 and LINC01234) and miRCURY LNA miRNA PCR (miR-625-5p), $n=3$. c Total RNA was extracted from A2058 cells transiently transfected with the indicated GapmeR for $48 \mathrm{~h}$ prior to generation of $\mathrm{CDNA}$ and analysis of PKM2 expression via qPCR, $n=3$. d TCGA-melanoma datasets were analysed to determine the impact of LINC00511 CNV amplification on PKM2 expression e Total RNA was extracted from A2058 cells transiently co-transfected with the indicated GapmeR alongside either miR-625-5p-specific miRCURY LNA power inhibitor or control for $48 \mathrm{~h}$ prior to generation of cDNA and analysis of PKM2 expression via qPCR, $n=3$. f A2058 cells were transiently co-transfected with the indicated GapmeR alongside either miR-625-5pspecific miRCURY LNA power inhibitor or control for $48 \mathrm{~h}$ prior to analysis of cellular respiration using the Cell Mito Stress Test kit, $n=3$.

We observed a downregulation of EMX2OS in several melanoma cell lines, compared with PM (Fig. S5). Moreover, analysis of LMC patient data demonstrated an inverse relationship between EMX2OS expression and tumour thickness, mitotic rate, and survival (Fig. S5), suggesting that this IncRNA may function as a tumour suppressor in melanoma. In contrast, A2058-specific SFPQenriched IncRNA included LINC00511, DUXAP8, TMEM51-AS1, FOXD2-AS1 and LINC01234, all of which have been shown to drive metastasis in a range of cancers via various mechanisms $[29,30,51-55]$, however, only FOXD2-AS1 has been associated previously with melanoma [54].

Our analysis of two SFPQ-enriched IncRNAs, LINC00511 and LINC01234, both with established roles as oncogenes in other cancer backgrounds, revealed that knockdown of these genes impaired melanoma viable cell growth, migration and, in the case of LINC00511, cellular respiration (Fig. 3b-f). Recently, LINC00511 was reported to accumulate in both nuclear and cytoplasmic fractions [33], suggesting that this IncRNA may possess sponging activity. We demonstrated that LINC00511 expression is inversely correlated with miR-625-5p transcript levels (Fig. 4b) and that disruption of the LINC00511/miR-625-5p axis resulted in decreased expression of PKM2 (Fig. 4c-e), presumably via its well-defined role in regulating glycolytic flux [56]. PKM2 was absent from the list of SFPQ-enriched transcripts in our RIP-seq data and SFPQ knockdown had no impact on PKM expression or exon 9-10 splicing (Fig. 4c and Fig. S2), suggesting that it does not regulate alternative splicing of PKM. This result was somewhat unexpected, as SFPQ knockdown does reduce the expression of LINC00511 (Fig. 5a). This observation may relate to partial knockdown of SFPQ protein $(\sim 50 \%)$ via ASO-transfection, which resulted in an $\sim 40 \%$ decrease in LINC00511 transcript levels (Fig. 5a) compared with the $\sim 97 \%$ knockdown achieved by directly targeting LINC00511 with 
a

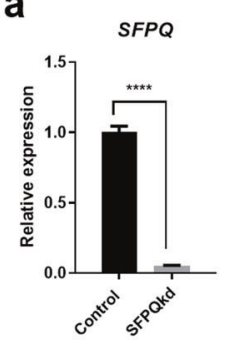

LINC00511

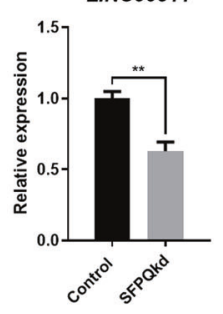

b

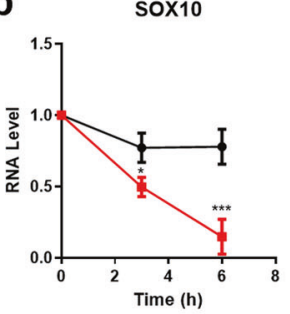

TBP

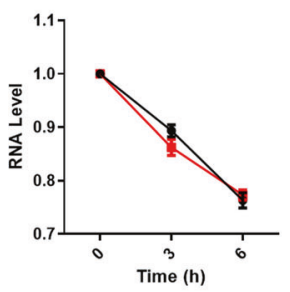

sox10

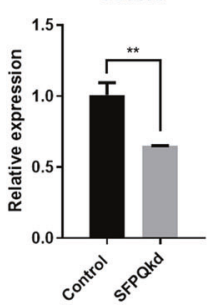

LINC01234

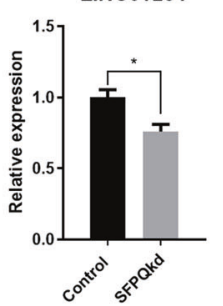

AMIGO2

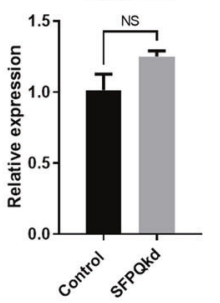

TMEM51-AS1

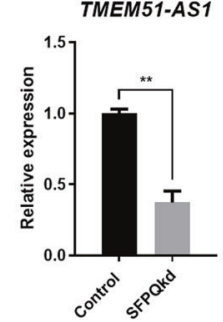

MAGEA3
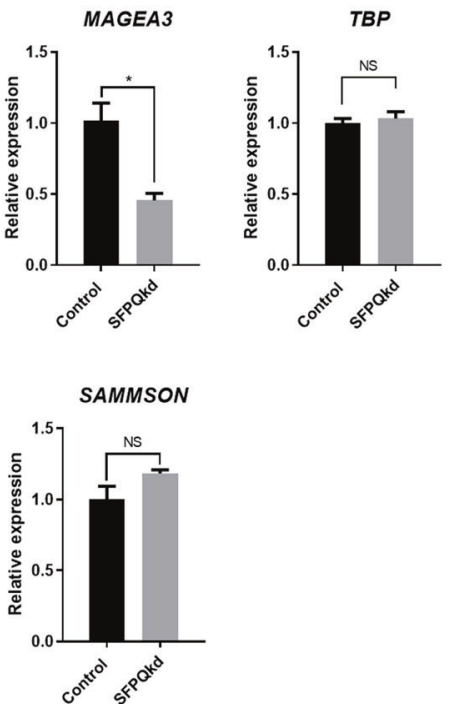

AMIGO2

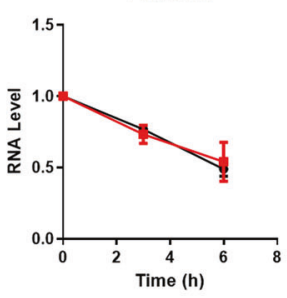

LINC00511

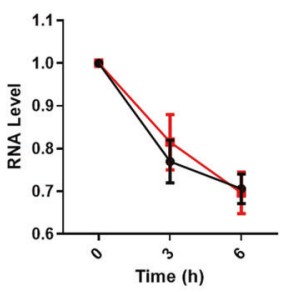

C

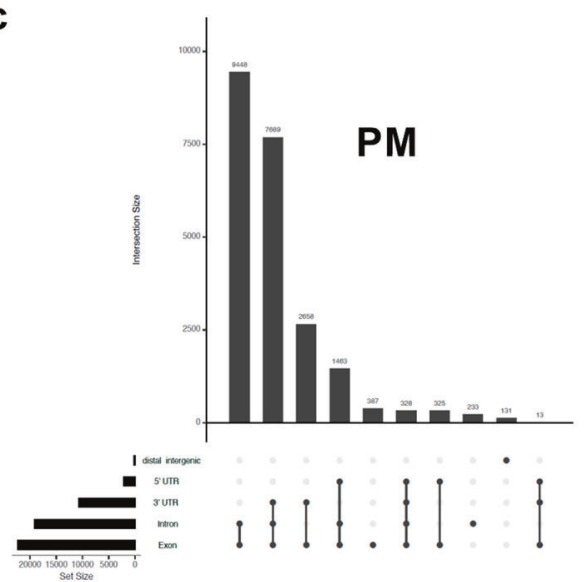

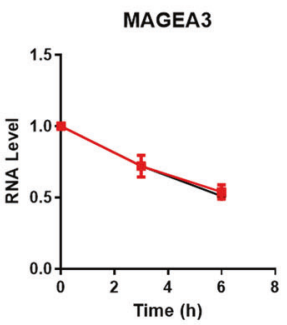

LINC01234

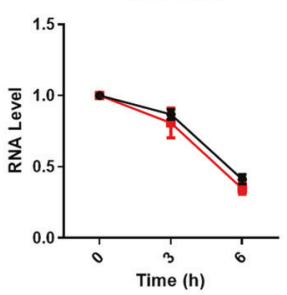

TMEM51-AS1

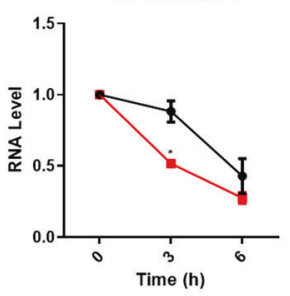

SAMMSON

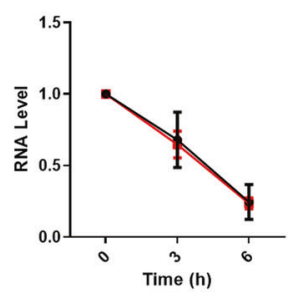

- Control

- SFPQkd

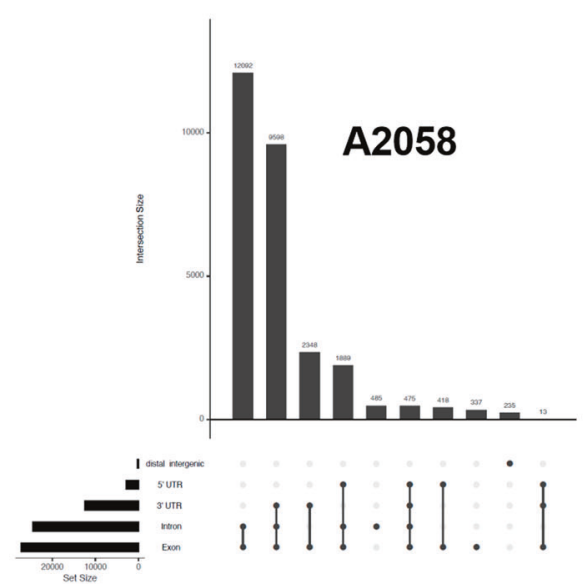

Fig. 5 SFPQ enhances the expression of target RNAs. a Total RNA was extracted from A2058 cells transiently transfected with SFPQ-specific GapmeR or control for $48 \mathrm{~h}$ prior to generation of CDNA and analysis of target gene expression via qPCR, $n=3$. b A2058 cells were transiently transfected with SFPQ-specific GapmeR or control for $48 \mathrm{~h}$ prior to the addition of actinomycin D $(1 \mu \mathrm{g} / \mathrm{ml})$. Total RNA was isolated from treated cells at 3 and $6 \mathrm{~h}$, cDNA generated and used to analyse target genes via qPCR, $n=3$. c ChIPseeker R package was used to annotate SFPQ enrichment on exon, intron, UTR, intergenic and promoter regions and data processed via the Integrative Genomics Viewer (IGV) to visualise the coverage of the reads across the identified genes. 
a

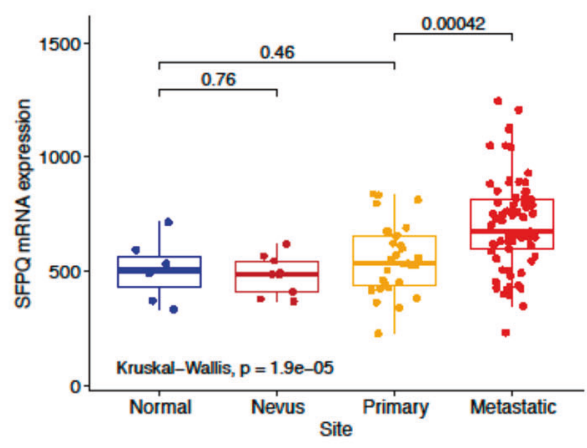

C

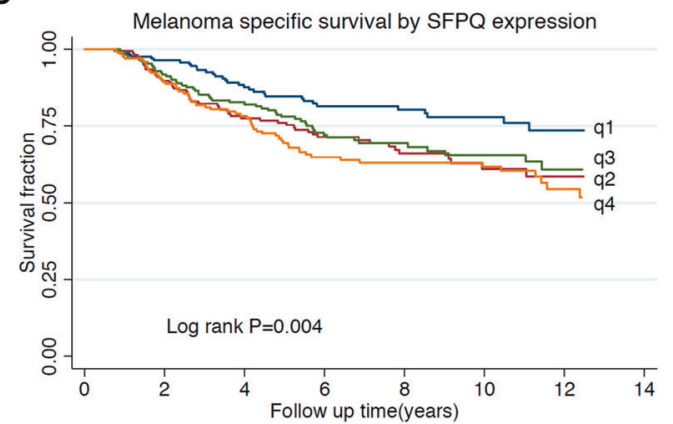

b

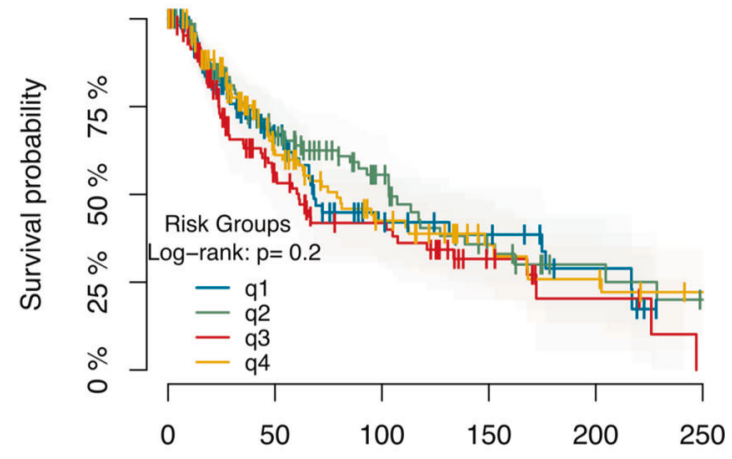

Survival Months

d
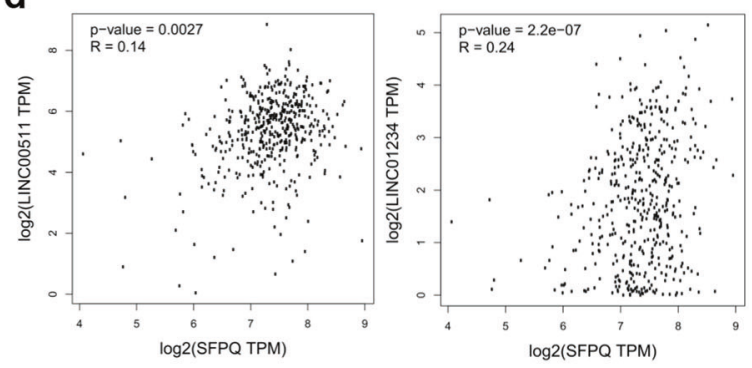

Fig. 6 Increased expression of SFPQ in primary melanoma is associated with decreased melanoma patient survival. a GEO data (GSE46517) was analysed to determine SFPQ mRNA expression in normal skin, benign nevi, primary and metastatic melanoma. b SFPQ expression did not associate with patient survival in TCGA-melanoma datasets. c Patients with lower SFPQ expression in primary tumours have an improved melanoma-specific survival in the LMC. Data presented in quartiles, q1-q4. d Expression correlation was analysed for SFPQ, LINC00511 and LINC01234 using TCGA-melanoma datasets on the GEPIA2 web portal.

ASOs (Fig. 4b). Therefore, it seems possible that while SFPQ knockdown leads to a significant decrease in the expression of LINC00511, this is not sufficient to dysregulate the LINC00511/miR625-5p axis and thus does not impact on PKM2 expression.

Our observation that melanoma-specific SFPQ-enriched genes were significantly decreased following SFPQ knockdown (Fig. 5a) and increased in cells overexpressing FLAG-SFPQ (Fig. S3) supports a role for SFPQ in efficient expression of bound-transcripts, rather than endogenous post-transcriptional processing events. Indeed, a recent article demonstrating that diminished availability of splicing factors in the nucleoplasm attenuates transcript release from the parent gene [57] provides a model whereby SFPQ may positively contribute to target RNA expression, a hypothesis that is particularly appealing given that SFPQ is a key regulator of transcriptional termination [58]. A second model, that is not mutually exclusive, is that SFPQ may regulate the expression of bound RNAs via increased stabilisation of the transcript. SFPQ has been previously shown to regulate the stability of $A R$ transcripts in prostate cancer and neurons and our own analysis of SFPQ-enriched transcripts identify SOX10 and the IncRNA, TMEM51-AS1 as being destabilised in SFPQ depleted cells (Fig. 5b). How SFPQ mediates transcript stability remains unclear, but one possibility is that SFPQ shields bound RNAs from degradation, in a manner analogous to other RNA-binding proteins (RBP), such as Human antigen $\mathrm{R}(\mathrm{HuR})[59,60]$ or the La-related protein superfamily [61, 62].

Analysis of a large population-based melanoma cohort demonstrated that melanoma patients with increased expression of SFPQ in primary tumours were significantly more likely to die due to their disease (Fig. 6c). However, while SFPQ expression was significantly increased in metastatic melanoma, compared with primary tumours (Fig. 6a), analysis of TCGA-melanoma datasets showed that differential expression of SFPQ in metastatic disease was not associated with patient survival (Fig. 6b). There are several differences between the LMC and TCGA cohorts. The most obvious is that LMC tumours are all primaries at different AJCC stages ( $35 \%$ stage I, 50\% stage II and $15 \%$ stage III) while TCGA's are mostly metastases (>80\%). Moreover, LMC samples are population based (patients were recruited as they came into clinic), while TCGA samples are selected. Finally, in the LMC cohort cause of death and subsequently analysed survival was exclusive to melanoma-associated deaths. Thus, the different results observed in the LMC and TCGA datasets suggest that high expression of SFPQ in primary tumours might be a driver of disease progression and thus have some utility as a prognostic biomarker, but at late-stage disease, further increases in SFPQ expression are less significant.

In summary, we have shown that SFPQ positively contributes to the cancer phenotype in melanoma, likely via reprograming of the SFPQ-RNA interactome in melanoma to favour the expression of oncogenic transcripts. It will be interesting to determine the global impact of SFPQ knockout on RNA expression in melanoma, particularly given recent work in renal cancer demonstrating that RBPs have a wide-ranging impact on RNA expression programs and by extension the cancer transcriptome [63].

\section{MATERIALS AND METHODS}

\section{Tissue culture}

Immortalised cell lines were obtained from European Collection of Authenticated Cell Cultures and certified mycoplasma-free. UACC-62 and A375 cells were maintained in Minimum Essential Medium, M14 in Roswell Park Memorial Institute 1640 Medium (RPMI), and A2058 cells in Dulbecco's Modified Eagle Medium, in each case supplemented with $10 \%$ foetal bovine serum, $100 \mathrm{U} / \mathrm{ml}$ of penicillin and $100 \mu \mathrm{g} / \mathrm{ml}$ of streptomycin. Primary human melanocytes (CELLnTEC, Switzerland) were grown in a 2:1 
mixture of keratinocyte serum-free media (K-SFM) and Eagle's minimal essential medium supplemented with $25 \mu \mathrm{g} / \mathrm{ml}$ bovine pituitary extract, $0.2 \mathrm{ng} / \mathrm{ml} \mathrm{rEGF}$, penicillin $(100 \mathrm{U} / \mathrm{ml})$, streptomycin $(100 \mu \mathrm{g} / \mathrm{ml})$ and $2 \mathrm{mM} \mathrm{L-}$ glutamine. All cells were cultured at $37^{\circ} \mathrm{C}$ and $5 \% \mathrm{CO}_{2}$. LNA inhibitors were transfected into cells using HiPerfect (QIAgen, UK), as previously described [64]. Details of LNA inhibitors used in this study can be found in Table S1, pSFPQ-FLAG has been previously described [65].

\section{Cell migration}

For wound healing assays scratches were generated in a confluent cell sheet using a $200 \mu \mathrm{l}$ pipette tip and cells cultured in SFM. Images of each wound were captured over $24 \mathrm{~h}$ (EVOS XL Core Cell Imaging System, 4x objective) and wound closure measured using the MRI tool (Image J). Transwell migration assays were carried out in $8 \mu \mathrm{m}$ inserts (Starstedt, Germany) and migrated cells stained using $0.2 \%(\mathrm{w} / \mathrm{v})$ crystal violet (Fisher Science, UK), imaged (EVOS XL Core Cell Imaging System) and the number of migrated cells calculated, as previously described [66].

\section{Viable cell growth}

Cells were seeded at a density of $1 \times 10^{4}$ cells/well in white-walled 96-well plates (ThermoFisher, UK). After $24 \mathrm{~h}$, media was replaced with $100 \mu \mathrm{l}$ of SFM and cells incubated at $37^{\circ} \mathrm{C}$ for a further 24,48 and $72 \mathrm{~h}$ with viable cell growth assessed at each time point using CellTiterGlo Luminescent Cell Viability Assay (Promega, UK) and luminescence recorded $10 \mathrm{~min}$ after reagent addition using the GloMax ${ }^{\circledR}$ Explorer system (Promega, UK).

\section{Apoptosis}

Apoptosis and cell death was analysed via FACS using the Guava ${ }^{\circledR}$ easyCyte ${ }^{\text {TM }}$ Flow Cytometer (Luminex, US), as described elsewhere [67]. For analysis of caspase $3 / 7$ activity, cells were seeded at a density of $1 \times 10^{4}$ cells/well, cultured for $24 \mathrm{~h}$ prior to addition of $100 \mu \mathrm{l}$ of Caspase-Glo ${ }^{\otimes} 3 / 7$ reagent directly to cells, incubation for $1 \mathrm{~h}$ at RT and recording of luminescence using the GloMax ${ }^{\circledR}$ Explorer system (Promega, UK).

\section{Analysis of cellular respiration}

OCR was determined using the Mito Stress Test Kit and XFe96 Extracellular Flux Analyzer (Seahorse Bioscience, Billerica, MA, USA). Cells were seeded in Seahorse XF96 V3 PS Cell Culture Microplates (Agilent Technologies, UK). Following incubation, cells were transferred to unbuffered XF assay media at pH 7.4 supplemented with $25 \mathrm{mM}$ glucose and $1 \mathrm{mM}$ sodium pyruvate and placed at $37^{\circ} \mathrm{C}$ in a non- $\mathrm{CO}_{2}$ incubator for one hour prior to assay. Cells were analysed using the Seahorse XF Cell Mito Stress Test (Agilent Technologies, UK). Immediately following each run cells were lysed, and protein concentration determined by Sulforhodamine B assay for normalisation. OCR was automatically calculated and analysed by the Seahorse Wave software (Agilent Technologies, UK).

\section{qRT-PCR}

Total RNA was extracted from cells and $500 \mathrm{ng}$ used to generate CDNA prior to qRT-PCR analysis using SsoAdvanced SYBR master mix (Bio-Rad, UK) on the CFX96 system (Bio-Rad, UK) normalised against TBP, RPS13 and GAPDH reference genes, as described elsewhere (66). RNA stability assays were carried out using $1 \mu \mathrm{g} / \mathrm{ml}$ actinomycin-D, as previously described [68]. Micro-RNA expression was analysed using miRCURY LNA miRNA PCR assays (Qiagen, UK) and data normalised against SNORD44 and SNORD48 reference genes. A list of oligonucleotides used during this study can be found in Table S2.

\section{RIP-seq}

SFPQ-RNA immunoprecipitations were performed using SFPQ-specific antibody or IgG control (Table S3) and the Magna RIP ${ }^{T M}$ RNA-Binding Protein Immunoprecipitation Kit (Merck Millipore, USA). Total RNA libraries were prepared using TruSeq Stranded Total RNA Sample Prep Kit (Illumina, USA) and the TruSeq CDNA libraries were analysed via NextSeq 500 (Illumina, USA) and data deposited at GEA (GSE171291). Details of RIP-seq data analysis are available in Supplementary Methods.

\section{Cell dependency analysis}

Gene dependency data for 18120 across 54 Melanoma cell lines were download from DepMap project (https://depmap.org/portal/). DEMETER2 inferred z-scores representing cell viability independent gene knockdown effects and expression values were extracted for SFPQ.

\section{Clinical data}

The LMC transcriptomic data comprises 703 primaries profiled on Illumina DASL array HT12.4 (EGAS00001002922, [36, 69]). Correlation between tumour characteristics and tumour expression of targets was performed via Spearman rank correlation. Data from TCGA-SKCM [70] were analysed to determine the correlation between gene expression, copy number alteration and patient clinical characteristics. These expressions were further tested for association with melanoma-specific survival after their split into quartiles by applying Cox proportional hazards regression and plotting Kaplan-Meier survival curves. Where appropriate, certain quartiles were combined to reduce the number of parameters. Both single gene models and bivariate models were applied with an interaction term to test the independence between genes. These analyses were conducted in STATA v14 (StataCorp, TX, USA), or R using tools provided on the GEPIA2 web portal http://gepia2.cancer-pku.cn/\#survival.

\section{Statistical analysis}

Except otherwise stated, graphical data shown represent mean \pm standard error of mean (SEM) using at least three independent experiments. Differences between means was analysed by Student's $t$ test Mann-Whitney Wilcoxon test, Kruskal-Wallis test, or one-way ANOVA as detailed in the figure legends. Survival analyses were conducted using the Cox proportional hazards regression and Kaplan-Meier graphs with logrank test. Statistics was considered significant at $p<0.05$.

\section{REFERENCES}

1. Garbe C, Leiter U. Melanoma epidemiology and trends. Clin Dermatol. 2009;27:3-9.

2. Ghosh P, Chin L. Genetics and genomics of melanoma. Expert Rev Dermatol. 2009;4:131.

3. Qiu T, Wang H, Wang Y, Zhang Y, Hui Q, Tao K. Identification of genes associated with melanoma metastasis. Kaohsiung J Med Sci. 2015;31:553-61.

4. Djulbegovic MB, Uversky VN. Expanding the understanding of the heterogeneous nature of melanoma with bioinformatics and disorder-based proteomics. Int J Biol Macromol. 2020;150:1281-93.

5. Bottini S, Hamouda-Tekaya N, Mategot R, Zaragosi LE, Audebert S, Pisano S, et al. Post-transcriptional gene silencing mediated by microRNAs is controlled by nucleoplasmic Sfpq. Nat Commun. 2017;8:1189.

6. Imamura K, Imamachi N, Akizuki G, Kumakura M, Kawaguchi A, Nagata K, et al. Long noncoding RNA NEAT1-dependent SFPQ relocation from promoter region to paraspeckle mediates IL8 expression upon immune stimuli. Mol Cell. 2014;53:393-406.

7. Jaafar L, Li Z, Li S, Dynan WS. SFPQ*NONO and XLF function separately and together to promote DNA double-strand break repair via canonical nonhomologous end joining. Nucleic Acids Res. 2017;45:1848-59.

8. Mora Gallardo C, Sánchez de Diego A, Gutiérrez Hernández J, Talavera-Gutiérrez A, Fischer T, Martínez-A C, et al. Dido3-dependent SFPQ recruitment maintains efficiency in mammalian alternative splicing. Nucleic Acids Res. 2019;47:5381-94.

9. Rajesh C, Baker DK, Pierce AJ, Pittman DL. The splicing-factor related protein SFPQ/PSF interacts with RAD51D and is necessary for homology-directed repair and sister chromatid cohesion. Nucleic Acids Res. 2011;39:132-45.

10. Ke YD, Dramiga J, Schütz U, Kril JJ, Ittner LM, Schröder H, et al. Tau-mediated nuclear depletion and cytoplasmic accumulation of SFPQ in Alzheimer's and Pick's disease. PLoS ONE. 2012;7:e35678.

11. Lu J, Shu R, Zhu Y. Dysregulation and dislocation of SFPQ disturbed DNA organization in alzheimer's disease and frontotemporal dementia. J Alzheimers Dis. 2018;61:1311-21.

12. Luisier R, Tyzack GE, Hall CE, Mitchell JS, Devine $H$, Taha DM, et al. Intron retention and nuclear loss of SFPQ are molecular hallmarks of ALS. Nat Commun. 2018;9:2010.

13. Ji Q, Zhang L, Liu X, Zhou L, Wang W, Han Z, et al. Long non-coding RNA MALAT1 promotes tumour growth and metastasis in colorectal cancer through binding to SFPQ and releasing oncogene PTBP2 from SFPQ/PTBP2 complex. Br J Cancer. 2014;111:736-48.

14. Hu Z, Dong L, Li S, Li Z, Qiao Y, Li Y, et al. Splicing regulator p54(nrb) /non-POU domain-containing octamer-binding protein enhances carcinogenesis through oncogenic isoform switch of MYC box-dependent interacting protein 1 in hepatocellular carcinoma. Hepatology. 2020;72:548-68.

15. Gao Z, Chen $M$, Tian X, Chen L, Chen $L$, Zheng $X$, et al. A novel human IncRNA SANT1 cis-regulates the expression of SLC47A2 by altering SFPQ/E2F1/HDAC1 binding to the promoter region in renal cell carcinoma. RNA Biol. 2019;16:940-9.

16. Zeng C, Liu S, Lu S, Yu X, Lai J, Wu Y, et al. The c-Myc-regulated IncRNA NEAT1 and paraspeckles modulate imatinib-induced apoptosis in CML cells. Mol Cancer. 2018;17:130. 
17. Takayama KI, Suzuki T, Fujimura T, Yamada $Y$, Takahashi S, Homma Y, et al. Dysregulation of spliceosome gene expression in advanced prostate cancer by RNA-binding protein PSF. Proc Natl Acad Sci USA. 2017;114:10461-6.

18. Ru Y, Chen XJ, Guo WZ, Gao SG, Qi YJ, Chen P, et al. NEAT1_2-SFPQ axis mediates cisplatin resistance in liver cancer cells in vitro. Onco Targets Ther. 2018:11:5695-702.

19. de Silva HC, Lin MZ, Phillips L, Martin JL, Baxter RC. IGFBP-3 interacts with NONO and SFPQ in PARP-dependent DNA damage repair in triple-negative breast cancer. Cell Mol Life Sci. 2019;76:2015-30.

20. Mitobe $Y$, lino K, Takayama KI, Ikeda K, Suzuki T, Aogi K, et al. PSF promotes ERpositive breast cancer progression via posttranscriptional regulation of ESR1 and SCFD2. Cancer Res. 2020;80:2230-42.

21. Pellarin I, Dall'acqua A, Gambelli A, Pellizzari I, D'andrea S, Sonego $M$, et al. Splicing factor proline- and glutamine-rich (SFPQ) protein regulates platinum response in ovarian cancer-modulating SRSF2 activity. Oncogene. 2020;39:4390-403.

22. Volders PJ, Verheggen K, Menschaert G, Vandepoele K, Martens L, Vandesompele J, et al. An update on LNCipedia: a database for annotated human IncRNA sequences. Nucleic Acids Res. 2015;43:4363-4.

23. Gupta RA, Shah N, Wang KC, Kim J, Horlings HM, Wong DJ, et al. Long non-coding RNA HOTAIR reprograms chromatin state to promote cancer metastasis. Nature. 2010:464:1071-6.

24. Hua JT, Ahmed M, Guo H, Zhang Y, Chen S, Soares F, et al. Risk SNP-mediated promoter-enhancer switching drives prostate cancer through IncRNA PCAT19. Cell. 2018;174:564-75.e518.

25. Wang Z, Yang B, Zhang M, Guo W, Wu Z, Wang Y, et al. IncRNA epigenetic landscape analysis identifies EPIC1 as an oncogenic IncRNA that Interacts with MYC and promotes cell-cycle progression in cancer. Cancer Cell. 2018;33:706-20. e709.

26. Tsherniak A, Vazquez F, Montgomery PG, Weir BA, Kryukov G, Cowley GS, et al. Defining a cancer dependency map. Cell. 2017;170:564-76.e516.

27. Chen XE, Chen P, Chen S, Lu J, Ma T, Shi G, et al. Long non-coding RNA FENDRR inhibits migration and invasion of cutaneous malignant melanoma cells. Biosci Rep. 2020;40:40.

28. Gu Y, Feng C, Liu T, Zhang B, Yang L. The downregulation of IncRNA EMX2OS might independently predict shorter recurrence-free survival of classical papillary thyroid cancer. PLoS One. 2018;13:e0209338.

29. Sun CC, Li SJ, Li G, Hua RX, Zhou XH, Li DJ. Long intergenic noncoding RNA 00511 acts as an oncogene in non-small-cell lung cancer by binding to EZH2 and suppressing p57. Mol Ther Nucleic Acids. 2016;5:e385.

30. Zhang J, Sui S, Wu H, Zhang J, Zhang X, Xu S, et al. The transcriptional landscape of IncRNAs reveals the oncogenic function of LINC00511 in ER-negative breast cancer. Cell Death Dis. 2019;10:599.

31. Zhu FY, Zhang SR, Wang LH, Wu WD, Zhao H. LINC00511 promotes the progression of non-small cell lung cancer through downregulating LATS2 and KLF2 by binding to EZH2 and LSD1. Eur Rev Med Pharmacol Sci. 2019;23:8377-90.

32. Li JH, Liu S, Zhou H, Qu LH, Yang JH. starBase v2.0: decoding miRNA-ceRNA, miRNA-ncRNA and protein-RNA interaction networks from large-scale CLIP-Seq data. Nucleic Acids Res. 2014;42:D92-97.

33. Chen Z, Wu H, Zhang Z, Li G, Liu B. LINC00511 accelerated the process of gastric cancer by targeting miR-625-5p/NFIX axis. Cancer Cell Int. 2019;19:351.

34. Deng H, Huang C, Wang $Y$, Jiang H, Peng S, Zhao X. LINC00511 promotes the malignant phenotype of clear cell renal cell carcinoma by sponging microRNA625 and thereby increasing cyclin D1 expression. Aging. 2019;11:5975-91.

35. Xue J, Zhang F. LncRNA LINC00511 plays an oncogenic role in lung adenocarcinoma by regulating PKM2 expression via sponging miR-625-5p. Thorac Cancer. 2020;11:2570-9.

36. Muralidhar S, Filia A, Nsengimana J, Poźniak J, O'shea SJ, Diaz JM, et al. Vitamin DVDR signaling inhibits Wnt/beta-catenin-mediated melanoma progression and promotes antitumor immunity. Cancer Res. 2019;79:5986-98.

37. Tang Z, Kang B, Li C, Chen T, Zhang Z. GEPIA2: an enhanced web server for largescale expression profiling and interactive analysis. Nucleic Acids Res. 2019;47: W556-W560.

38. Hu L, Tang J, Huang $X$, Zhang T, Feng X. Hypoxia exposure upregulates MALAT-1 and regulates the transcriptional activity of PTB-associated splicing factor in A549 lung adenocarcinoma cells. Oncol Lett. 2018;16:294-300.

39. Wu CF, Tan GH, Ma CC, Li L. The non-coding RNA Ilme23 drives the malignant property of human melanoma cells. J Genet Genomics. 2013;40:179-88.

40. Wesley UV, Albino AP, Tiwari S, Houghton AN. A role for dipeptidyl peptidase IV in suppressing the malignant phenotype of melanocytic cells. J Exp Med. 1999:190:311-22.

41. Fontanals-Cirera B, Hasson D, Vardabasso C, Di Micco R, Agrawal P, Chowdhury A, et al. Harnessing BET inhibitor sensitivity reveals AMIGO2 as a melanoma survival gene. Mol Cell. 2017;68:731-44.e9.
42. Graf SA, Busch C, Bosserhoff AK, Besch R, Berking C. SOX10 promotes melanoma cell invasion by regulating melanoma inhibitory activity. J Investig Dermatol. 2014;134:2212-20.

43. Roeder C, Schuler-Thurner B, Berchtold S, Vieth G, Driesch PV, Schuler G, et al. MAGE-A3 is a frequent tumor antigen of metastasized melanoma. Arch Dermatol Res. 2005;296:314-9.

44. Shakhova O, Zingg D, Schaefer SM, Hari L, Civenni G, Blunschi J, et al. Sox10 promotes the formation and maintenance of giant congenital naevi and melanoma. Nat Cell Biol. 2012;14:882-90.

45. Wang D, Wang J, Ding N, Li Y, Yang Y, Fang X, et al. MAGE-A1 promotes melanoma proliferation and migration through C-JUN activation. Biochem Biophys Res Commun. 2016;473:959-65.

46. Li Y, Zhang W, Liu P, Xu Y, Tang L, Chen W, et al. Long non-coding RNA FENDRR inhibits cell proliferation and is associated with good prognosis in breast cancer. Onco Targets Ther. 2018;11:1403-12.

47. Wang B, Xian J, Zang J, Xiao L, Li Y, Sha M, et al. Long non-coding RNA FENDRR inhibits proliferation and invasion of hepatocellular carcinoma by downregulating glypican-3 expression. Biochem Biophys Res Commun. 2019;509:143-7.

48. Zhang G, Han G, Zhang X, Yu Q, Li Z, Li Z, et al. Long non-coding RNA FENDRR reduces prostate cancer malignancy by competitively binding miR-18a-5p with RUNX1. Biomarkers. 2018:23:435-45.

49. Zhu Y, Zhang $X$, Wang L, Zhu X, Xia Z, Xu L, et al. FENDRR suppresses cervical cancer proliferation and invasion by targeting miR-15a/b-5p and regulating TUBA1A expression. Cancer Cell Int. 2020;20:152.

50. Duan $M$, Fang $M$, Wang $C$, Wang $H$, Li M. LncRNA EMX2OS induces proliferation, invasion and sphere formation of ovarian cancer cells via regulating the miR-6543p/AKT3/PD-L1 axis. Cancer Manag Res. 2020;12:2141-54.

51. Chen Y, Zhao H, Li H, Feng X, Tang H, Qiu C, et al. LINC01234/MicroRNA-31-5p/ MAGEA3 axis mediates the proliferation and chemoresistance of hepatocellular carcinoma cells. Mol Ther Nucleic Acids. 2020;19:168-78.

52. Hui L, Wang J, Zhang J, Long J. IncRNA TMEM51-AS1 and RUSC1-AS1 function as ceRNAs for induction of laryngeal squamous cell carcinoma and prediction of prognosis. PeerJ. 2019;7:e7456.

53. Lin MG, Hong YK, Zhang Y, Lin BB, He XJ. Mechanism of IncRNA DUXAP8 in promoting proliferation of bladder cancer cells by regulating PTEN. Eur Rev Med Pharmacol Sci. 2018;22:3370-7.

54. Ren W, Zhu Z, Wu L. FOXD2-AS1 correlates with the malignant status and regulates cell proliferation, migration, and invasion in cutaneous melanoma. J Cell Biochem. 2019;120:5417-23.

55. Zhao X, Liu Y, Li Z, Zheng S, Wang Z, Li W, et al. Linc00511 acts as a competing endogenous RNA to regulate VEGFA expression through sponging hsa-miR-29b$3 p$ in pancreatic ductal adenocarcinoma. J Cell Mol Med. 2018;22:655-67.

56. Christofk HR, Vander Heiden MG, Harris MH, Ramanathan A, Gerszten RE, Wei R, et al. The M2 splice isoform of pyruvate kinase is important for cancer metabolism and tumour growth. Nature. 2008;452:230-3.

57. Hochberg-Laufer H, Neufeld N, Brody Y, Nadav-Eliyahu S, Ben-Yishay R, Shav-Tal $Y$. Availability of splicing factors in the nucleoplasm can regulate the release of mRNA from the gene after transcription. PLoS Genet. 2019;15:e1008459.

58. Kaneko S, Rozenblatt-Rosen O, Meyerson M, Manley JL. The multifunctional protein p54nrb/PSF recruits the exonuclease XRN2 to facilitate pre-mRNA 3' processing and transcription termination. Genes Dev. 2007;21:1779-89.

59. Chai Y, Liu J, Zhang Z, Liu L. HuR-regulated IncRNA NEAT1 stability in tumorigenesis and progression of ovarian cancer. Cancer Med. 2016;5:1588-98.

60. Hu YP, Jin YP, Wu XS, Yang Y, Li YS, Li HF, et al. LncRNA-HGBC stabilized by HuR promotes gallbladder cancer progression by regulating miR-502-3p/SET/AKT axis. Mol Cancer. 2019;18:167.

61. Hopkins TG, Mura M, Al-Ashtal HA, Lahr RM, Abd-Latip N, Sweeney K, et al. The RNA-binding protein LARP1 is a post-transcriptional regulator of survival and tumorigenesis in ovarian cancer. Nucleic Acids Res. 2016;44:1227-46.

62. Shao R, Scully SJ Jr., Yan W, Bentley B, Mueller J, Brown C, et al. The novel lupus antigen related protein acheron enhances the development of human breast cancer. Int J Cancer. 2012;130:544-54.

63. Perron G, Jandaghi P, Solanki S, Safisamghabadi M, Storoz C, Karimzadeh M, et al. A general framework for interrogation of mRNA stability programs identifies RNA-binding proteins that govern cancer transcriptomes. Cell Rep. 2018;23:1639-50.

64. Anene C, Graham AM, Boyne J, Roberts W. Platelet microparticle delivered microRNA-Let-7a promotes the angiogenic switch. Biochim Biophys Acta Mol Basis Dis. 2018;1864:2633-43.

65. Snijders AP, Hautbergue GM, Bloom A, Williamson JC, Minshull TC, Phillips HL, et al. Arginine methylation and citrullination of splicing factor proline- and glutamine-rich (SFPQ/PSF) regulates its association with mRNA. RNA. 2015;21:347-59. 
66. Akhbari P, Tobin D, Poterlowicz K, Roberts W, Boyne JR. MCV-miR-M1 Targets the Host-Cell Immune Response Resulting in the Attenuation of Neutrophil Chemotaxis. J Investig Dermatol. 2018;138:2343-54.

67. Williamson AJ, Dibling BC, Boyne JR, Selby P, Burchill SA. Basic fibroblast growth factor-induced cell death is effected through sustained activation of p38MAPK and up-regulation of the death receptor p75NTR. J Biol Chem. 2004;279:47912-28.

68. Baquero-Perez B., Antanaviciute A., Yonchev I. D., Carr I. M., Wilson S. A., Whitehouse A. The Tudor SND1 protein is an m(6)A RNA reader essential for replication of Kaposi's sarcoma-associated herpesvirus. Elife. 2019;8:e47261.

69. Nsengimana J, Laye J, Filia A, O'shea S, Muralidhar S, Poźniak J, et al. betaCatenin-mediated immune evasion pathway frequently operates in primary cutaneous melanomas. J Clin Investig. 2018;128:2048-63.

70. Cancer Genome Atlas N. Genomic classification of cutaneous melanoma. Cell. 2015;161:1681-96.

\section{ACKNOWLEDGEMENTS}

The authors would like to thank Dr. M.J. Thornton and Dr. S. Sikkink for assistance with primary melanocyte cell culture and Prof. M. Dickman for providing the PSFPQFLAG construct. This work was funded by a personal scholarship to OB by Mr. Shaukat Ali, British Skin Foundation award to JRB (030/s/18) and MRC award MR/M019012/1 to JNB.

\section{COMPETING INTERESTS}

The authors declare no competing interests.

\section{ADDITIONAL INFORMATION}

Supplementary information The online version contains supplementary material available at https://doi.org/10.1038/s41388-021-01912-4.

Correspondence and requests for materials should be addressed to J.R.B.

Reprints and permission information is available at http://www.nature.com/ reprints

Publisher's note Springer Nature remains neutral with regard to jurisdictional claims in published maps and institutional affiliations.

(i) Open Access This article is licensed under a Creative Commons Attribution 4.0 International License, which permits use, sharing, adaptation, distribution and reproduction in any medium or format, as long as you give appropriate credit to the original author(s) and the source, provide a link to the Creative Commons license, and indicate if changes were made. The images or other third party material in this article are included in the article's Creative Commons license, unless indicated otherwise in a credit line to the material. If material is not included in the article's Creative Commons license and your intended use is not permitted by statutory regulation or exceeds the permitted use, you will need to obtain permission directly from the copyright holder. To view a copy of this license, visit http://creativecommons. org/licenses/by/4.0/.

(c) Crown 2021 